\title{
Per se Performance of Pumpkin Hybrids for Small Size, Thick Flesh with High Yield and Quality Traits
}

\author{
R. Kumar ${ }^{1 *}$, V. Rajasree ${ }^{1}$, S. Praneetha ${ }^{1}$, S. Rajeswari², \\ U. Tripura ${ }^{3}$ and V. S. Sriyamuna ${ }^{1}$ \\ ${ }^{1}$ Department of Vegetable Crops, Horticulture College and Research Institute, TNAU, \\ Coimbatore-641003, Tamil Nadu, India \\ ${ }^{2}$ Centre for Plant Breeding and Genetics, Agriculture College and Research Institute, TNAU, \\ Coimbatore-641003, Tamil Nadu, India \\ ${ }^{3}$ Department of Spice and Plantation crops, Horticulture College and Research Institute, \\ TNAU, Coimbatore-641003, Tamil Nadu, India \\ *Corresponding author
}

\section{A B S T R A C T}

An investigation was carried out to study the performance of 18 hybrids of pumpkin (Cucurbita moschata Duch. ex Poir) through Line x Tester mating design. Observations

\section{Keywords}

Small size,

Pumpkin, beta

carotene

Article Info

Accepted:

20 May 2018

Available Online:

10 June 2018 were recorded on the traits viz., vine length, days to first female flower appearance, node number for first female flower appearance, sex ratio, days to first harvest, fruit number per vine, fruit weight, flesh thickness and fruit yield per vine, besides quality traits such as ascorbic acid content, beta carotene content and crude fibre content in the fruit. Evaluation of hybrids for per se and sca revealed that the cross Rajasthan Local x Pusa Viswas $\left(\mathrm{L}_{5} \mathrm{x}\right.$ $\mathrm{T}_{2}$ ) was adjudged as the best hybrid, since it recorded the highest mean and sca effect for traits of study viz., fruit weight and total yield per vine. The next best hybrid, Ambili $\mathrm{x}$ Pusa Viswas $\left(\mathrm{L}_{2} \times \mathrm{T}_{2}\right)$ could also be justified as the best combination through less days for first female flower appearance, more fruit number per vine, crude fibre content and fruit yield per vine. The next best hybrid, Saras x Pusa Viswas $\left(\mathrm{L}_{3} \times \mathrm{T}_{2}\right)$ could also be adjusted as the best combination, since it recorded the highest mean and sca effect of traits of study like flesh thickness and beta carotene content. Thus, first generation hybrids can be wellutilized for exploiting hybrid vigour to achieve improved quality.

\section{Introduction}

Pumpkin (Cucurbita moschata Duch. ex Poir,) originated in Central Mexico and is cultivated in the tropical and subtropical regions of the world. It is an important cucurbitaceous vegetable crop of India, constituting a principal ingredient in several Indian dishes.
Pumpkin has received little attention in crop improvement compared to other cucurbitaceous vegetables even it is a rich source of beta carotene next to carrot. Vitamin A deficiency constitute a public health problem and affects mainly children and women. In India, consumers prefer dark yellow color, round shape fruit with thick and 
deep yellow internal flesh color. In pumpkin, the major problem is its large-sized fruits (4-5 $\mathrm{kg}$ each). This is not preferred by the present nuclear families of three to four members.

Further, with increase in number of such families recently in India, customers prefer to buy only whole fruits of medium-size pumpkins, instead of cut pieces. Further, small fruits are easily packed and transported, without any damage. Therefore, developing pumpkin hybrids with small sized fruits (1-2 $\mathrm{kg}$ ) with rich in beta carotene content is essential. The present study was undertaken to evaluate $\mathrm{F}_{1}$ hybrids for yield and quality purpose.

\section{Materials and Methods}

The investigation was carried out at Department of Vegetable Crops, Horticulture College and Research Institute, Tamil Nadu Agricultural University, Coimbatore, during 2016-17, with $18 \mathrm{~F}_{1}$ hybrids (obtained by crossing 6 lines and 3 testers through line $x$ tester mating design) along with the standard check (Arjuna) from East West Seeds (P) Ltd. Field experiments with the hybrids were laid out in Randomized Block Design, with two replications and five plants per replication at a spacing of $1.5 \times 2.0 \mathrm{~m}^{2}$. Recommended package of practices of TNAU was followed to grow a successful crop of pumpkin. Observations were recorded in five randomly selected plants in each replication on important quantitative traits, viz., vine length (m), days to first female flower appearance, node number for first female flower appearance, sex ratio, days to first harvest, fruit number per vine, fruit weight $(\mathrm{kg})$, flesh thickness $(\mathrm{cm})$ and fruit yield per vine $(\mathrm{kg})$ besides quality traits such as ascorbic acid content $(\mathrm{mg} / \mathrm{l00g})$, beta carotene content $(\mu \mathrm{g} / \mathrm{g})$ and crude fibre content of the fruit $(\%)$ were analysed as per AOAC method (1975), Chopra and Kanwar method (1972). Statistical analysis of data was done to estimate per se values and degree of significance of various traits (Panse and Sukhatme, 1978).

\section{Results and Discussion}

In Pumpkin hybrids exhibited significant differences for all the characters under study for growth, yield and quality, thus offering scope for selecting high-yielding hybrids with good quality traits. Results of per se performance of hybrids are presented in Tables 1. The sca effect of a hybrid denotes deviation from performance prediction based on gca of the parents (Allard, 1960). The sca effect seen is due to dominance, epistasis and environmental influence. Under certain favourable conditions, all the non-additive gene functions may be triggered and may result in high sca effect and mean value of a responding hybrid. Thus, evaluation of a hybrid for high per se and sca effect is also an important criterion. Hybrids with high per se and sca effect were evaluated for selecting the best hybrids.

The gca and sca values of parents and hybrids are presented in Tables 2 and 3, respectively. Vine length is an important parameter for obtaining high fruit yield in pumpkin. Among the 18 hybrids of pumpkin studied, the cross 'Odisha Local x CO2' followed by 'Ambili x Punjab Samrat' and 'Mysore Local x Punjab Samrat' exhibited high sca and mean performance for vine length. Sharma et al., (1993) recorded similar results in bitter gourd in the cross 'Pocha Seed x PSPL'.

In these crosses, the parents, Odisha Local, Ambili except Mysore Local (poor combiner) and the tester Punjab Samrat exhibited good general combing ability for vine length. A predominant role of non-additive gene action for vine length in pumpkin was reported by Tamilselvi et al., (2013), Sirohi and Ghorui (1993) and Nisha (1999). 
Days taken to first female flower appearance is considered as one of the essential criteria for selecting for earliness in hybrids. The present study of 18 pumpkin crosses, the $s c a$ variances of days taken to first female flower appearance were greater than those of gca suggesting the better role of non-additive genetic factors than that of additive action. It was also noticed that, the cross Ambili x $\mathrm{CO} 2$ $\left(\mathrm{L}_{2} \times \mathrm{T}_{3}\right)$, Ambili x Pusa Viswas $\left(\mathrm{L}_{2} \times \mathrm{T}_{2}\right)$ and Rajasthan Local x Punjab Samrat $\left(\mathrm{L}_{5} \times \mathrm{T}_{1}\right)$ exhibited the high negative and significant sca and less mean performance for earliness in terms of days for first female flower appearance. In these crosses, the parents Rajasthan Local $\left(\mathrm{L}_{5}\right)$, Ambili $\left(\mathrm{L}_{2}\right)$, and the tester Punjab Samrat $\left(\mathrm{T}_{1}\right)$ exhibited good general combing ability and high per se values for days for first female flower appearance. Neeraj Sharma et al., (2002) recorded the similar results in bottle gourd. The lowest mean performance for sex ratio was observed in the hybrids Odisha Local x CO2 $\left(\mathrm{L}_{4} \times \mathrm{T}_{3}\right)$ followed by Ambili x Punjab Samrat $\left(\mathrm{L}_{2} \times \mathrm{T}_{1}\right)$ among the 18 pumpkin hybrids of the study while, the hybrids Ambili x Punjab Samrat $\left(\mathrm{L}_{2}\right.$ $\mathrm{x} \mathrm{T}_{1}$ ) followed by Odisha Local $\mathrm{x}$ Punjab Samrat $\left(\mathrm{L}_{4} \times \mathrm{T}_{1}\right)$ recorded lowest mean coupled with negative significant $s c a$ values for the same trait. Kumaresan et al., (2005) also endorsed the same results of lowest sex ratio by the snake gourd hybrid PKM-1 $\mathrm{x}$ Mahyco Short. In the present investigation, the variance due to sca was greater than that of gca for sex ratio which referred the preponderance of non-additive gene action for sex ratio was clearly indicated.

The fruit number per vine has a considerable influence on yield of fruits. The hybrids Saras $\mathrm{x}$ Pusa Viswas $\left(\mathrm{L}_{3} \times \mathrm{T}_{2}\right)$ followed by Ambili $\mathrm{x}$ Pusa Viswas $\left(\mathrm{L}_{2} \times \mathrm{T}_{2}\right)$ recorded the highest per se coupled with significant $s c a$ effect for fruit number per vine. In this cross, as the female parents Ambili $\left(\mathrm{L}_{2}\right)$ had already proved as a good general combiner for this trait.
Similar results were observed in bitter gourd, by Lawande and Patil (1989) where the hybrid Green Long x CO2 gave higher significant sca value for fruit number per vine. In the present investigation, the variance due to sca was much greater than the gca indicating the influence of non-additive gene action for fruit number per vine. This is in accordance with the reports of Richard Kennedy (1994), Munshi and Sirohi (1994) in bitter gourd that non-additive gene action had influenced this trait. With respect to fruit weight, the pumpkin crosses Rajasthan Local x Pusa Viswas $\left(\mathrm{L}_{5} \mathrm{x}\right.$ $\mathrm{T}_{2}$ ) followed by Odisha Local $\mathrm{x}$ Pusa Viswas $\left(\mathrm{L}_{4} \times \mathrm{T}_{2}\right)$ recorded the highest mean and $s c a$ effect. In these crosses, the parents Rajasthan Local $\left(\mathrm{L}_{5}\right)$, Odisha Local $\left(\mathrm{L}_{4}\right)$, and the tester Pusa Viswas $\left(\mathrm{T}_{2}\right)$ exhibited good general combing ability and high per se values for same trait.

Similar results were recorded by Rao et al., (2000) in ridge gourd. In pumpkin, flesh thickness is an important parameter. In this study, the hybrids Rajasthan Local x CO2 $\left(\mathrm{L}_{5}\right.$ $\left.\mathrm{x} \quad \mathrm{T}_{3}\right)$ and Saras $\mathrm{x}$ Pusa Viswas $\left(\mathrm{L}_{3} \times \mathrm{T}_{2}\right)$ recorded the highest per se coupled with significant sca effect for fruit flesh thickness.

In these crosses, the parents Rajasthan Local $\left(\mathrm{L}_{5}\right)$ and the tester Pusa Viswas $\left(\mathrm{T}_{2}\right)$ exhibited good general combing ability and high per se values for same trait. This is in agreement with the findings of Nisha (1999) in pumpkin.

It was observed that seed weight was governed by dominant gene or non-additive gene action. The hybrid Mysore Local x Pusa Viswas $\left(\mathrm{L}_{1} \times \mathrm{T}_{2}\right)$ followed by Saras x CO2 $\left(\mathrm{L}_{3}\right.$ $\mathrm{x} \mathrm{T}_{3}$ ) recorded the better significant mean and $s c a$ values. These results were coincides with the results obtained by Munshi and Sirohi (1994) and Richard Kennedy (1994) in bitter gourd and Nisha (1999) in pumkin that the hybrid $\mathrm{CM} 31$ x $\mathrm{CO} 2$ recorded the highest mean and sca values for this trait. 
Table.1 Per se performance of hybrids for different traits in Pumpkin

\begin{tabular}{|c|c|c|c|c|c|c|c|c|c|c|c|c|c|c|c|c|c|}
\hline Hybrids & 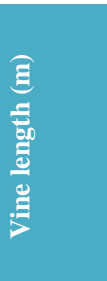 & 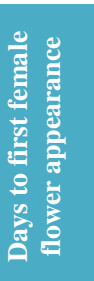 & 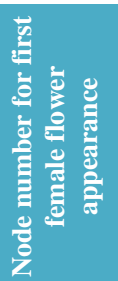 & 赵 & 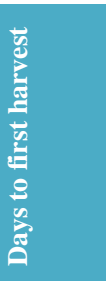 & 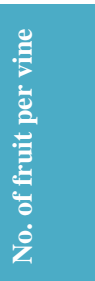 & 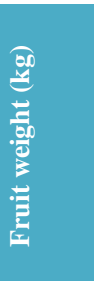 & 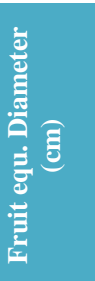 & 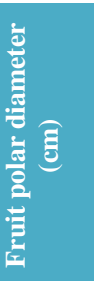 & 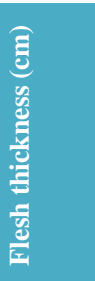 & 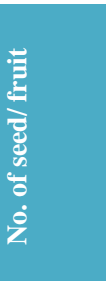 & 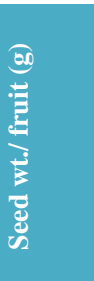 & 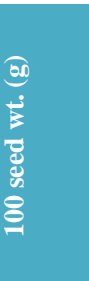 & 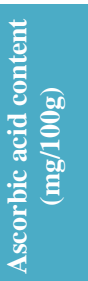 & 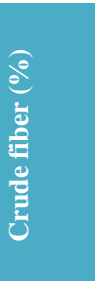 & 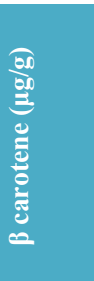 & 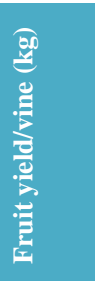 \\
\hline $\mathrm{L}_{1} \times \mathrm{T}_{1}$ & 4.52 & 51.10 & 7.90 & 18.26 & 90.33 & 4.20 & 1.21 & 16.00 & 16.50 & 2.30 & 210.00 & 27.30 & 13.00 & 5.32 & 0.98 & 40.32 & 7.22 \\
\hline $\mathrm{L}_{1} \times \mathrm{T}_{2}$ & 3.48 & 53.40 & 15.70 & 15.11 & 98.20 & 4.80 & 1.72 & 17.20 & 11.50 & 2.10 & 280.00 & 29.60 & 10.57 & 6.30 & 1.04 & 25.46 & 11.09 \\
\hline $\mathrm{L}_{1} \times \mathrm{T}_{3}$ & 3.89 & 53.80 & 7.40 & 16.70 & 120.30 & 4.30 & 2.31 & 17.40 & 15.00 & 3.20 & 140.00 & 24.80 & 17.71 & 4.20 & 1.06 & 24.45 & 7.31 \\
\hline $\mathrm{L}_{2} \times \mathrm{T}_{1}$ & 4.94 & 51.70 & 8.25 & 14.19 & 98.70 & 5.30 & 1.70 & 16.50 & 9.00 & 2.50 & 310.01 & 15.60 & 5.03 & 6.29 & 0.86 & 12.44 & 5.83 \\
\hline $\mathrm{L}_{2} \times \mathrm{T}_{2}$ & 4.30 & 42.20 & 8.03 & 14.84 & 88.20 & 6.80 & 1.10 & 15.50 & 10.00 & 2.10 & 163.00 & 12.30 & 7.55 & 6.34 & 1.30 & 28.06 & 14.03 \\
\hline $\mathbf{L}_{2} \times T_{3}$ & 4.10 & 43.10 & 7.70 & 15.79 & 95.31 & 4.40 & 2.50 & 22.50 & 10.40 & 2.70 & 280.00 & 21.20 & 7.57 & 5.31 & 0.94 & 17.46 & 10.38 \\
\hline $\mathrm{L}_{3} \times \mathrm{T}_{1}$ & 3.22 & 51.60 & 12.90 & 23.88 & 94.60 & 2.10 & 1.96 & 14.20 & 12.00 & 3.10 & 312.00 & 20.20 & 6.47 & 7.20 & 1.20 & 14.16 & 5.38 \\
\hline $\mathrm{L}_{3} \times \mathrm{T}_{2}$ & 3.86 & 51.90 & 11.80 & 15.23 & 88.89 & 6.50 & 1.70 & 17.50 & 14.00 & 3.80 & 188.00 & 15.30 & 8.14 & 8.31 & 1.45 & 89.60 & 10.10 \\
\hline $\mathrm{L}_{3} \times \mathrm{T}_{3}$ & 3.56 & 50.90 & 12.80 & 21.45 & 93.14 & 3.10 & 1.14 & 16.00 & 12.50 & 1.20 & 230.00 & 26.80 & 11.65 & 6.20 & 1.05 & 9.61 & 3.53 \\
\hline $\mathbf{L}_{4} \times T_{1}$ & 4.92 & 52.70 & 12.20 & 14.20 & 83.10 & 5.23 & 2.16 & 19.30 & 12.00 & 3.00 & 328.00 & 26.30 & 8.02 & 6.30 & 1.23 & 2.54 & 11.30 \\
\hline $\mathbf{L}_{4} \times \mathbf{T}_{2}$ & 4.28 & 47.70 & 6.10 & 14.77 & 86.90 & 5.10 & 2.75 & 20.60 & 16.00 & 4.10 & 258.00 & 24.30 & 9.42 & 8.80 & 1.86 & 4.59 & 12.29 \\
\hline $\mathbf{L}_{4} \times \mathbf{T}_{3}$ & 4.98 & 52.80 & 14.90 & 13.35 & 94.60 & 5.30 & 2.07 & 18.10 & 10.00 & 3.90 & 356.00 & 29.30 & 8.23 & 6.20 & 1.45 & 5.44 & 10.97 \\
\hline $\mathrm{L}_{5} \times \mathrm{T}_{1}$ & 3.89 & 45.80 & 9.90 & 19.23 & 82.62 & 3.28 & 1.90 & 19.50 & 12.50 & 2.70 & 326.00 & 22.60 & 6.93 & 5.89 & 0.84 & 17.45 & 6.23 \\
\hline $\mathbf{L}_{5} \times \mathbf{T}_{2}$ & 4.20 & 50.30 & 12.60 & 14.63 & 83.81 & 5.90 & 3.24 & 19.50 & 18.30 & 3.10 & 278.00 & 20.30 & 7.30 & 9.20 & 0.93 & 16.26 & 16.65 \\
\hline $\mathrm{L}_{5} \times \mathrm{T}_{3}$ & 4.09 & 50.40 & 12.50 & 14.64 & 95.81 & 5.13 & 2.07 & 20.30 & 11.50 & 3.90 & 280.00 & 25.60 & 9.14 & 8.71 & 1.08 & 20.30 & 10.62 \\
\hline $\mathrm{L}_{6} \times \mathrm{T}_{1}$ & 3.26 & 52.60 & 6.20 & 22.56 & 98.32 & 2.36 & 1.27 & 15.50 & 8.20 & 2.60 & 288.00 & 15.60 & 5.42 & 3.20 & 1.01 & 21.14 & 3.00 \\
\hline $\mathrm{L}_{6} \times \mathrm{T}_{2}$ & 3.89 & 63.00 & 14.30 & 23.12 & 102.08 & 2.16 & 1.55 & 16.90 & 12.20 & 2.10 & 138.00 & 13.20 & 9.57 & 4.82 & 0.95 & 9.95 & 3.35 \\
\hline $\mathrm{L}_{6} \times \mathrm{T}_{3}$ & 3.64 & 63.30 & 3.50 & 23.64 & 115.30 & 2.12 & 1.26 & 15.80 & 9.30 & 2.00 & 182.00 & 20.68 & 11.36 & 2.09 & 0.39 & 10.97 & 2.67 \\
\hline Check Hybrid & 4.89 & 69.00 & 22.10 & 19.27 & 115.80 & 4.16 & 3.20 & 20.80 & 12.00 & 3.40 & 395.22 & 24.20 & 6.22 & 9.93 & 2.93 & 18.53 & 8.74 \\
\hline Mean & 4.06 & 51.57 & 10.26 & 17.53 & 95.01 & 4.34 & 1.87 & 17.68 & 12.27 & 2.80 & 252.61 & 21.72 & 9.06 & 6.15 & 1.09 & 20.57 & 8.44 \\
\hline SEd & 0.050 & 0.718 & 0.149 & 0.238 & 1.213 & 0.056 & 0.027 & 0.183 & 0.189 & 0.034 & 3.064 & 0.335 & 0.086 & 0.079 & 0.017 & 0.486 & 0.105 \\
\hline C.D. $(0.05)$ & 0.105 & 1.546 & 0.315 & 0.501 & 2.556 & 0.119 & 0.572 & 0.386 & 0.389 & 0.072 & 6.465 & 0.707 & 0.182 & 0.168 & 0.036 & 1.024 & 0.222 \\
\hline
\end{tabular}

Mysore Local $\left(\mathrm{L}_{1}\right)$, Ambili $\left(\mathrm{L}_{2}\right)$, Saras $\left(\mathrm{L}_{3}\right)$, Odisha Local $\left(\mathrm{L}_{4}\right)$, Rajasthan Local $\left(\mathrm{L}_{5}\right), \mathrm{CO} 1\left(\mathrm{~L}_{6}\right)$, Punjab Samrat $\left(\mathrm{T}_{1}\right)$, Pusa Viswas $\left(\mathrm{T}_{2}\right), \mathrm{CO} 2\left(\mathrm{~T}_{3}\right)$ 
Table.2 General combining ability effects of pumpkin parents

\begin{tabular}{|c|c|c|c|c|c|c|c|c|c|c|c|c|c|c|c|c|c|}
\hline 苞 & 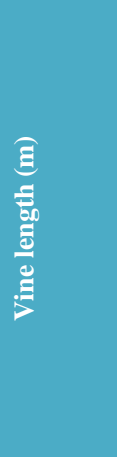 & 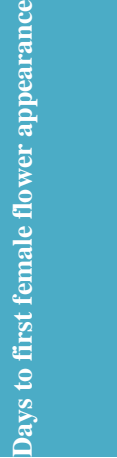 & 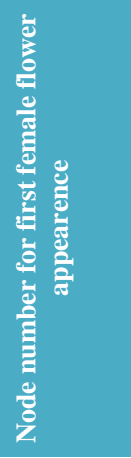 & 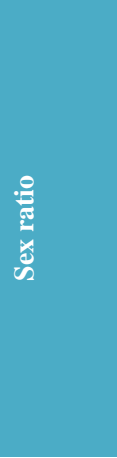 & 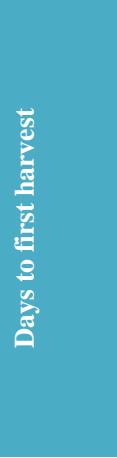 & 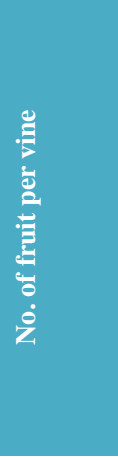 & 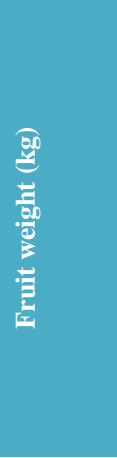 & 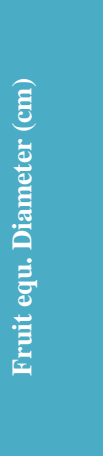 & 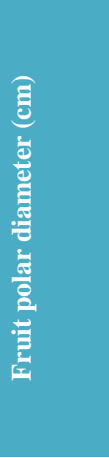 & 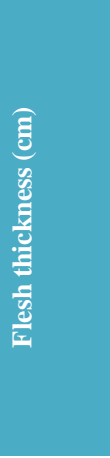 & 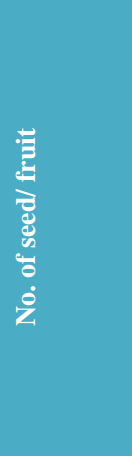 & 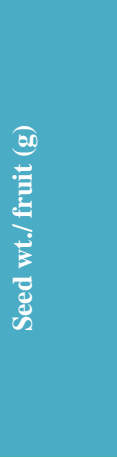 & 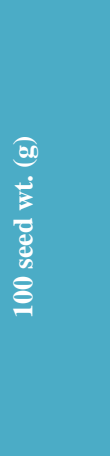 & 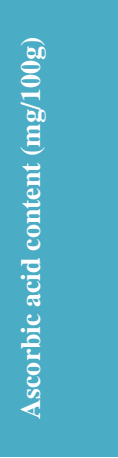 & 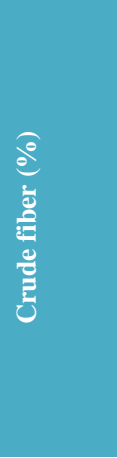 & 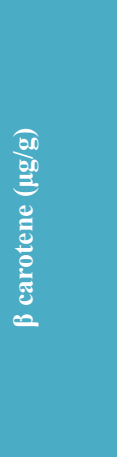 & 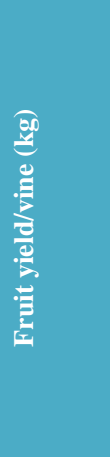 \\
\hline $\mathbf{L}_{1}$ & $-0.09 * *$ & $1.19 * *$ & $0.07 \mathrm{~ns}$ & $-0.84 * *$ & $7.93 * *$ & $0.10 * *$ & $-0.12 * *$ & -0.82 ** & $2.06 * *$ & $-0.27 * *$ & $-42.61 * *$ & $5.51 * *$ & 4.70 ** & -0.88 ** & $-0.06 * *$ & $9.51 * *$ & $0.10 *$ \\
\hline $\mathbf{L}_{2}$ & $0.39 * *$ & $-5.91 * *$ & $-2.27 * *$ & $-2.59 * *$ & $-0.94 \mathrm{~ns}$ & 1.16 ** & $-0.10 * *$ & $0.48 * *$ & $-2.47 * *$ & -0.37 ** & $-1.61 \mathrm{~ns}$ & $-5.35 * *$ & $-2.35 * *$ & $-0.17 * *$ & $-0.06^{* *}$ & $-1.25 * *$ & $1.64 * *$ \\
\hline $\mathbf{L}_{3}$ & -0.51 ** & $-0.11 \mathrm{~ns}$ & $2.24 * *$ & $2.65 * *$ & $-2.80 * *$ & -0.44 ** & -0.27 ** & -1.78 ** & 0.56 ** & -0.10 ** & $-9.28 * *$ & $-0.95 * *$ & $-0.31 * *$ & 1.09 ** & 0.14 ** & $17.22 * *$ & -2.10 ** \\
\hline $\mathbf{L}_{4}$ & 0.67 ** & $-0.51 \mathrm{~ns}$ & $0.81 * *$ & $-3.43 * *$ & $-6.81 * *$ & $0.87 * *$ & $0.46 * *$ & 1.65 ** & $0.39 * *$ & $0.87 * *$ & 61.39 ** & $4.91 * *$ & $-0.50 * *$ & 0.95 ** & $0.42 * *$ & $-16.38 * *$ & $3.08 * *$ \\
\hline $\mathbf{L}_{5}$ & $0.00 \mathrm{~ns}$ & $-2.74 * *$ & $1.41 * *$ & $-1.37 * *$ & $-7.60 * *$ & $0.43 * *$ & $0.54 * *$ & 2.08 ** & $1.83 * *$ & $0.43 * *$ & 42.05 ** & $1.11 * *$ & $-1.27 * *$ & $1.78 * *$ & $-0.14 * *$ & $-2.56 * *$ & $2.73 * *$ \\
\hline $\mathrm{L}_{6}$ & $-0.46 * *$ & $8.06 * *$ & $-2.26 * *$ & $5.57 * *$ & $10.22 * *$ & $-2.12 * *$ & $-0.51 * *$ & $-1.62 * *$ & $-2.37 * *$ & -0.57 ** & $-49.95 * *$ & $-5.23 * *$ & $-0.28 * *$ & $-2.78 * *$ & $-0.31 * *$ & $-6.55 * *$ & $-5.44 * *$ \\
\hline SEd & 0.020 & 0.293 & 0.061 & 0.970 & 0.495 & 0.023 & 0.011 & 0.075 & 0.077 & 0.014 & 1.251 & 0.137 & 0.035 & 0.032 & 0.007 & 0.198 & 0.043 \\
\hline$T_{1}$ & $0.07 * *$ & $-0.66 * *$ & $-0.70 * *$ & $1.19 * *$ & $-3.73 * *$ & $-0.59 * *$ & $-0.17 * *$ & -0.85 ** & $-0.57 * *$ & $-0.10 * *$ & $43.06 * *$ & $-0.45 * *$ & $-1.58 * *$ & $-0.45 * *$ & $-0.07 * *$ & $-2.56 * *$ & $-1.95 * *$ \\
\hline$T_{2}$ & $-0.06 * *$ & $-0.16 \mathrm{~ns}$ & $1.16 * *$ & $-1.25 * *$ & $-3.66 * *$ & $0.87 * *$ & $0.14 * *$ & 0.18 ** & $1.39 * *$ & $0.08 * *$ & $-35.11 * *$ & $-2.55 * *$ & $-0.30 * *$ & $1.15 * *$ & $0.16 * *$ & $8.42 * *$ & $2.81 * *$ \\
\hline$T_{3}$ & $-0.01 \mathrm{~ns}$ & $0.81 * *$ & $-0.46 * *$ & $0.06 \mathrm{~ns}$ & $7.40 * *$ & $-0.28 * *$ & $0.02 * *$ & $0.67 * *$ & $-0.82 * *$ & $0.02 \mathrm{~ns}$ & $-7.95 * *$ & $3.01 * *$ & $1.88 * *$ & $-0.70 * *$ & $-0.10 * *$ & $-5.86 * *$ & $-0.86 * *$ \\
\hline SEd & 0.014 & 0.207 & 0.043 & 0.069 & 0.350 & 0.016 & 0.008 & 0.053 & 0.055 & 0.010 & 0.884 & 0.097 & 0.025 & 0.023 & 0.005 & 0.140 & 0.030 \\
\hline
\end{tabular}


Table.3 Specific combining ability effects of hybrids for different traits in pumpkin

\begin{tabular}{|c|c|c|c|c|c|c|c|c|c|c|c|c|c|c|c|c|c|}
\hline Hybrids & 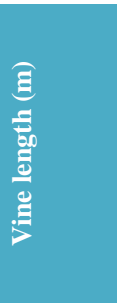 & 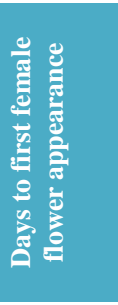 & 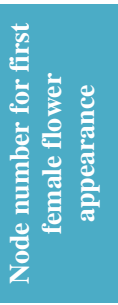 & 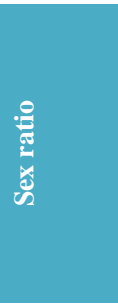 & 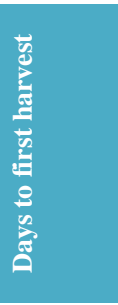 & 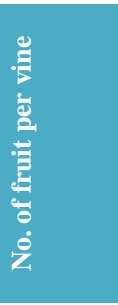 & 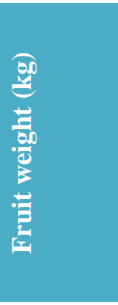 & 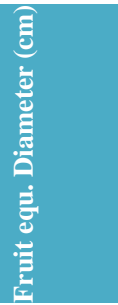 & 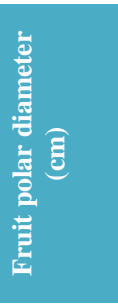 & 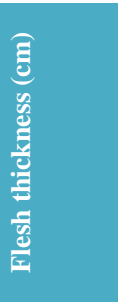 & 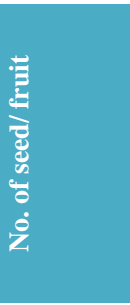 & 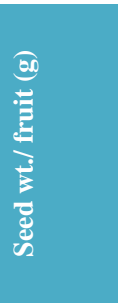 & 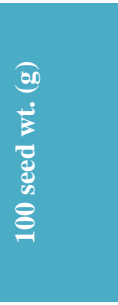 & 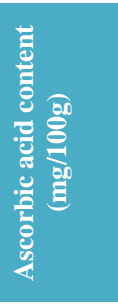 & 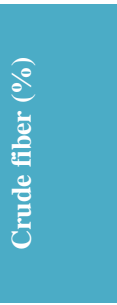 & 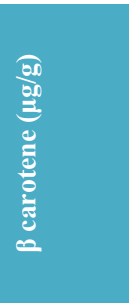 & 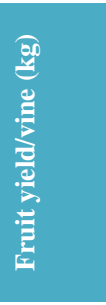 \\
\hline $\mathrm{L}_{1} \times \mathrm{T}_{1}$ & $0.49 * *$ & $-1.01 \mathrm{NS}$ & $-1.73 * *$ & $0.38 *$ & $-8.88 * *$ & $0.36 * *$ & $-0.37 * *$ & $-0.02 \mathrm{NS}$ & $2.74 * *$ & $-0.13 * *$ & $-43.05 * *$ & $0.52 *$ & $0.82 * *$ & $0.50 * *$ & $0.02 \mathrm{~ns}$ & $12.80 * *$ & $0.63 * *$ \\
\hline$\overline{L_{1} \times T_{2}}$ & $-0.43 * *$ & $0.79 \mathrm{NS}$ & $4.20 * *$ & $-0.33 \mathrm{NS}$ & $-1.08 \mathrm{NS}$ & $-0.51 * *$ & $-0.17 * *$ & $0.15 \mathrm{NS}$ & $-4.23 * *$ & $-0.52 * *$ & $105.11 * *$ & $4.92 * *$ & $-2.89 * *$ & $-0.12 *$ & $-0.15 * *$ & $-13.04 * *$ & $-0.26 * *$ \\
\hline$L_{1} \times T_{3}$ & $-0.06 \mathrm{NS}$ & $0.22 \mathrm{NS}$ & $-2.47 * *$ & $-0.05 \mathrm{NS}$ & $9.96 * *$ & $0.15 * *$ & $0.54 * *$ & $-0.13 \mathrm{NS}$ & $1.49 * *$ & $0.65 * *$ & $-62.06 * *$ & $-5.44 * *$ & $2.07 * *$ & $-0.38 * *$ & $0.13 * *$ & $0.24 \mathrm{NS}$ & $-0.37 * *$ \\
\hline $\mathrm{L}_{2} \times \mathrm{T}_{1}$ & $0.42 * *$ & $6.69 * *$ & $0.96 * *$ & $-1.94 * *$ & $8.36 * *$ & $0.39 * *$ & $0.10 * *$ & $-0.82 * *$ & $-0.23 \mathrm{NS}$ & $0.17 * *$ & $15.95 * *$ & $-0.31 \mathrm{NS}$ & $-0.10 \mathrm{NS}$ & $0.76 * *$ & $-0.10 * *$ & $-4.32 * *$ & $-2.30 * *$ \\
\hline $\mathrm{L}_{2} \times \mathrm{T}_{2}$ & $-0.09 *$ & $-3.31 * *$ & $-1.13 * *$ & $1.15 * *$ & $-2.21 *$ & $0.43 * *$ & $-0.81 * *$ & $-2.85 * *$ & $-1.19 * *$ & $-0.42 * *$ & $-52.89 * *$ & $-1.51 * *$ & $1.13 * *$ & $-0.79 * *$ & $0.10 * *$ & $0.32 \mathrm{NS}$ & $1.14 * *$ \\
\hline $\mathbf{L}_{2} \times \mathrm{T}_{3}$ & $-0.33 * *$ & $-3.38 * *$ & $0.17 \mathrm{NS}$ & $0.79 * *$ & $-6.16 * *$ & $-0.82 * *$ & $0.71 * *$ & $3.67 * *$ & $1.42 * *$ & $0.25 * *$ & $36.94 * *$ & $1.82 * *$ & $-1.03 * *$ & $0.03 \mathrm{~ns}$ & $0.00 \mathrm{~ns}$ & $4.00 * *$ & $1.16 * *$ \\
\hline$\overline{L_{3} \times T_{1}}$ & $-0.39 * *$ & $0.79 \mathrm{NS}$ & $1.10 * *$ & $2.51 * *$ & $6.12 * *$ & $-1.21 * *$ & $0.53 * *$ & $-0.85 * *$ & $-0.26 \mathrm{NS}$ & $0.50 * *$ & $25.61 * *$ & $-0.11 \mathrm{NS}$ & $-0.70 * *$ & $0.41 * *$ & $0.04 * *$ & $-21.07 * *$ & $0.99 * *$ \\
\hline $\mathrm{L}_{3} \times \mathrm{T}_{2}$ & $0.37 * *$ & $0.59 \mathrm{NS}$ & $-1.86 * *$ & $-3.71 * *$ & $0.35 \mathrm{NS}$ & $1.73 * *$ & $-0.04 *$ & $1.42 * *$ & $-0.23 \mathrm{NS}$ & $1.02 * *$ & $-20.22 * *$ & $-2.91 * *$ & $-0.31 * *$ & $-0.07 \mathrm{NS}$ & $0.05 * *$ & $43.39 * *$ & $0.95 * *$ \\
\hline $\mathrm{L}_{3} \times \mathrm{T}_{3}$ & $0.03 \mathrm{NS}$ & $-1.38 *$ & $0.76 * *$ & $1.20 * *$ & $-6.47 * *$ & $-0.52 * *$ & $-0.48 * *$ & $-0.57 * *$ & $0.49 * *$ & $-1.52 * *$ & $-5.39 *$ & $3.02 * *$ & $1.01 * *$ & $-0.34 * *$ & $-0.09 * *$ & $-22.32 * *$ & $-1.94 * *$ \\
\hline$\overline{\mathrm{L}_{4} \times \mathrm{T}_{1}}$ & $0.12 * *$ & $2.29 * *$ & $1.83 * *$ & $-1.09 * *$ & $-1.37 \mathrm{NS}$ & $0.61 * *$ & $0.00 \mathrm{NS}$ & $0.82 * *$ & $-0.09 \mathrm{NS}$ & $-0.57 * *$ & $-29.06 * *$ & $0.12 \mathrm{NS}$ & $1.04 * *$ & $-0.35 * *$ & $-0.21 * *$ & $0.91 *$ & $1.73 * *$ \\
\hline $\mathrm{L}_{4} \times \mathrm{T}_{2}$ & $-0.39 * *$ & $-3.21 * *$ & $-6.13 * *$ & $1.91 * *$ & $2.37 *$ & $-0.98 * *$ & $0.28 * *$ & $1.08 * *$ & $1.94 * *$ & $0.35 * *$ & $-20.89 * *$ & $0.22 \mathrm{NS}$ & $1.17 * *$ & $0.56 * *$ & $0.18 * *$ & $-8.02 * *$ & $-2.04 * *$ \\
\hline $\mathrm{L}_{4} \times \mathrm{T}_{3}$ & $0.27 * *$ & $0.92 \mathrm{NS}$ & $4.29 * *$ & $-0.82 * *$ & $-1.00 \mathrm{NS}$ & $0.37 * *$ & $-0.28 * *$ & $-1.90 * *$ & $-1.84 * *$ & $0.22 * *$ & $49.94 * *$ & $-0.34 \mathrm{NS}$ & $-2.21 * *$ & $-0.20 * *$ & $0.03 *$ & $7.11 * *$ & $0.31 * *$ \\
\hline $\mathrm{L}_{5} \times \mathrm{T}_{1}$ & $-0.24 * *$ & $-2.38 * *$ & $-1.06 * *$ & $1.88 * *$ & $-1.06 \mathrm{NS}$ & $-0.90 * *$ & $-0.34 * *$ & $0.58 * *$ & $-1.03 * *$ & $-0.43 * *$ & $-11.72 * *$ & $0.22 \mathrm{NS}$ & $0.72 * *$ & $-1.59 * *$ & $-0.04 * *$ & $2.00 * *$ & $-2.99 * *$ \\
\hline $\mathrm{L}_{5} \times \mathrm{T}_{2}$ & $0.19 * *$ & $1.62 * *$ & $-0.23 *$ & $-0.29 \mathrm{NS}$ & $0.06 \mathrm{NS}$ & $0.26 * *$ & $0.69 * *$ & $-0.45 * *$ & $2.81 * *$ & $-0.22 * *$ & $18.44 * *$ & $0.02 \mathrm{NS}$ & $-0.19 * *$ & $0.12 *$ & $-0.19 * *$ & $-10.16 * *$ & $2.67 * *$ \\
\hline $\mathrm{L}_{5} \times \mathrm{T}_{3}$ & $0.04 \mathrm{NS}$ & $0.76 \mathrm{NS}$ & $1.29 * *$ & $-1.59 * *$ & $1.00 \mathrm{NS}$ & $0.64 * *$ & $-0.36 * *$ & $-0.13 \mathrm{NS}$ & $-1.78 * *$ & $0.65 * *$ & $-6.72 * *$ & $-0.24 \mathrm{NS}$ & $-0.53 * *$ & $1.47 * *$ & $0.23 * *$ & 8.16 ** & 0.31 ** \\
\hline$\overline{L_{6} \times T_{1}}$ & $-0.41 * *$ & $-6.38 * *$ & $-1.10 * *$ & $-1.73 * *$ & $-3.18 * *$ & $0.74 * *$ & $0.08 * *$ & $0.28 *$ & $-1.13 * *$ & $0.47 * *$ & $42.28 * *$ & $-0.44 \mathrm{NS}$ & $-1.78 * *$ & $0.28 * *$ & $0.30 * *$ & $9.68 * *$ & $1.94 * *$ \\
\hline $\mathrm{L}_{6} \times \mathrm{T}_{2}$ & $0.35 * *$ & $3.52 * *$ & $5.14 * *$ & $1.26 * *$ & $0.51 \mathrm{NS}$ & $-0.93 * *$ & $0.05 *$ & $0.65 * *$ & $0.91 * *$ & $-0.22 * *$ & $-29.56 * *$ & $-0.74 * *$ & $1.08 * *$ & $0.30 * *$ & $0.00 \mathrm{NS}$ & $-12.49 * *$ & $-2.46 * *$ \\
\hline $\mathrm{L}_{6} \times \mathrm{T}_{3}$ & $0.06 \mathrm{NS}$ & $2.86 * *$ & $-4.04 * *$ & $0.47 *$ & $2.67 * *$ & $0.19 * *$ & $-0.12 * *$ & $-0.93 * *$ & $0.22 \mathrm{NS}$ & $-0.25 * *$ & $-12.72 * *$ & $1.18 * *$ & 0.70 ** & $-0.58 * *$ & $-0.30 * *$ & $2.81 * *$ & $0.53 * *$ \\
\hline$\overline{\text { SEd }}$ & 0.0351 & 0.5076 & 0.1057 & 0.168 & 0.8574 & 0.0398 & 0.0192 & 0.1294 & 0.1335 & 0.024 & 2.1664 & 0.2368 & 0.0609 & 0.0561 & 0.0119 & 0.3433 & 0.0743 \\
\hline
\end{tabular}

*Significant at 5 per cent level * * Significant at 1 per cent level

Mysore Local $\left(\mathrm{L}_{1}\right)$, Ambili $\left(\mathrm{L}_{2}\right)$, Saras $\left(\mathrm{L}_{3}\right)$, Odisha Local $\left(\mathrm{L}_{4}\right)$, Rajasthan Local $\left(\mathrm{L}_{5}\right), \mathrm{CO} 1\left(\mathrm{~L}_{6}\right)$, Punjab Samrat $\left(\mathrm{T}_{1}\right)$, Pusa Viswas $\left(\mathrm{T}_{2}\right)$, CO2 $\left(\mathrm{T}_{3}\right)$ 
With respect to quality parameters, the highest per se and sca values of beta carotene content was observed in Saras x Pusa Viswas $\left(\mathrm{L}_{3} \times \mathrm{T}_{2}\right)$ followed by Mysore Local x Punjab Samrat $\left(\mathrm{L}_{1} \times \mathrm{T}_{1}\right)$ proved its good performance in developing hybrids with high carotene content as proved through the significant mean, gca and sca performance. Hazara et al., (2007) endorsed the same results in pumpkin. In case of crude fibre content though the crosses Odisha Local x Pusa Viswas $\left(\mathrm{L}_{4} \times \mathrm{T}_{2}\right)$ and Ambili x Pusa Viswas $\left(\mathrm{L}_{2} \times \mathrm{T}_{2}\right)$ were found to be the best crosses to develop hybrids with high total crude fibre content as adjudged by their mean per se values and sca values. Similar results were observed in ridge gourd, by Shivanand Hegde (2009) where the hybrid IC 393014 x IC 413592 gave highest significant mean value for crude fibre content. Expression of yield to its fullest potential is the prime important trait to be considered in hybridization programme. Based on the per se performance and sca of hybrids, the crosses Rajasthan Local x Pusa Viswas $\left(\mathrm{L}_{5} \times \mathrm{T}_{2}\right)$ followed by Ambili x Pusa Viswas $\left(\mathrm{L}_{2} \times \mathrm{T}_{2}\right)$ proved to be the best specific combiners for yield which proved their superiority through gca and sca values. Choudhary et al., (2006) also justified similar results that the crosses MS1 x Punjab Sunheri and MS1 x Hara Madhu exhibited the highest sca effect and recorded the highest fruit yield per vine in muskmelon.

In conclusion, evaluation of hybrids for per se and sca revealed that the cross Rajasthan Local $x$ Pusa Viswas $\left(\mathrm{L}_{5} \times \mathrm{T}_{2}\right)$ was adjudged as the best hybrid, since it recorded the highest mean and sca effect for traits of study $v i z$, fruit weight and total yield per vine. The next best hybrid, Ambili x Pusa Viswas $\left(\mathrm{L}_{2} \mathrm{X}\right.$ $\mathrm{T}_{2}$ ) could also be justified as the best combination through less days for first female flower appearance, more fruit number per vine, crude fibre content and fruit yield per vine. The another best hybrid, Saras x Pusa
Viswas $\left(\mathrm{L}_{3} \times \mathrm{T}_{2}\right)$ could also be adjusted as the best combination, since it recorded the highest mean and sca effect of traits of study like flesh thickness and beta carotene content.

\section{References}

Allard, R.W. 1960. Principles of plant breeding. John Wiley and Sons Inc., New York, USA.

Chopra, R. and S.L. Kanwar. 1976. Analytical Agricultural Chemistry. Kalyani Publishers New Delhi, India p: 36.

Choudhary, B.R., M.S. Fageria, Sudhakar Pandey and Mathura Rai. 2006. Combining ability studies for economic attributes in muskmelon (Cucumis melo L.). Veg. Sci., 33: 185-187.

Hazara, P., A.K. Mandal, A.K. Dutta, D. Sikadar and M.K. Pandit. 2007. Breeding Pumpkin (Cucurbita moschata Duch. ex Poir.) for High Yield and Carotene Content. Proc. I IC on Indig. Veg. and Legumes Eds. M.L. Chadha et al., Acta Hort. 752: 431-435.

Kumaresan, G.R., S. Makesh and N. Ramaswamy. 2005. Combining ability studies for yield and its components in Snake gourd. Crop Res., 31(1): 103106.

Lawande, K.E. and A.V. Patil. 1991. Studies on gene action in bitter gourd (Momordica charantia L.). Veg. Sci., 18(2): 192-199.

Munshi, A.D. and P.S. Sirohi. 1994. Combining ability estimates in bitter gourd (Momordica charantia L.). Veg. Sci., 21(2): 132-136.

Neeraj Sharma, N.K. Sharma and Y.S. Malik. 2002. Combining ability in long fruited bottle gourd. Haryana J. Hort. Sci., 31: 79-82.

Nisha, S.K. 1999. Genetic studies in pumpkin (Cucurhita moschata Duch. ex Poir.) through diallele analysis. M.Sc. (Hort.) 
Thesis, Tamil Nadu Agri. Univ., Coimbatore, TN, India.

Panse, V.G. and P.V. Sukhatme. 1978. Statistical methods for agricultural workers ICAR, New Delhi, India.

Rao, B.N., P.V. Rao and Y.N. Reddy. 2000. Combining ability studies in ridge gourd [Luffa aculangula (Roxb.) L.]. Intl. J. Tropical Agri., 18: 141-146.

Richard Kennedy, R. 1994. Line x Tester analysis in bitter gourd. M.Sc. (Hort.) Thesis. Tamil Nadu Agric. Univ., Coimbatore.

Sharma, N.K., B.S. Dhankhar and A.S. Tewaria. 1993. Line x Tester analysis for combining ability studies in bottle gourd - A note. Haryana J. Hort. Sci., 22: 324-327.

Shivanand Hegde. 2009. Studies on heterosis in ridge gourd. M.Sc. Thesis, Tamil Nadu Agri. Univ., Coimbatore, TN, India.

Sirohi, P.S. and S. Ghorui. 1993. Gene effects of certain quantitative characters in pumpkin. Veg. Sci., 20: 158- 162.

Tamilselvi, N.A., P. Jansirani and L. Pugalendhi. 2013. Evaluation of $\mathrm{F}_{1}$ hybrids of pumpkin (Cucurbita moschata Duch ex Poir.) for yield and quality. J. Hort. Sci., 8(2): 187-194.

\section{How to cite this article:}

Kumar R., V. Rajasree, S. Praneetha, S. Rajeswari, U. Tripura and Sriyamuna V. S. 2018. Per se Performance of Pumpkin Hybrids for Small Size, Thick Flesh with High Yield and Quality Traits. Int.J.Curr.Microbiol.App.Sci. 7(06): 2591-2598.

doi: https://doi.org/10.20546/ijcmas.2018.706.306 